\title{
Developing and expanding hospital antimicrobial stewardship: The Ontario experience
}

\author{
Nakamachi $Y^{1,2}$, West $S^{1}$, Dresser $L^{1,3}$, Morris $A M^{1,4^{*}}$ \\ ${ }^{1}$ Mount Sinai Hospital, University Health Network, Toronto, ON \\ ${ }^{2}$ Department of Pharmacy, University Health Network, Toronto, ON \\ ${ }^{3}$ Leslie Dan Faculty of Pharmacy, University of Toronto, Toronto, ON \\ ${ }^{4}$ Mount Sinai Hospital, University Health Network, and University of Toronto, Toronto, ON
}

*Correspondence: amorris@mtsinai.on.ca

\begin{abstract}
Mount Sinai Hospital and University Health Network, two academic health science centres in Toronto, Ontario, jointly established a robust, well-resourced antimicrobial stewardship program (ASP). Over the course of four years, we spread our program to five intensive care units (ICUs), learned which change management practices worked and which did not, and leveraged our ICU successes to other areas of our hospitals. We identified the following two factors as critical to establishing ASPs in hospitals: strong leadership with clear accountability; and valid, reliable data to monitor progress. Subsequently we have led the spread of our program to 14 academic hospital ICUs, and more recently we leveraged to help community hospitals implement ASPs without in-house infectious diseases specialists. We introduced three new data fields into the provincial critical care information system: days of antibacterial therapy, days of antifungal therapy, and ICU-onset $C$. difficile, which will help standardize data collection moving forward. This model—starting with academic health sciences centres, and antimicrobial stewardship experts and leaders who are then supported to mentor and develop new experts and leaders-could be copied in other jurisdictions both within and outside of Canada.
\end{abstract}

\section{Introduction}

Antimicrobial stewardship is a programmatic effort aimed at optimizing the appropriate use of antimicrobials (1). In this article, we will describe the Mount Sinai Hospital-University Health Network Antimicrobial Stewardship Program involvement in the spread of hospital-based antimicrobial stewardship in three phases: 1) Academic Hospital Foundation; 2) Academic Hospital Spread; 3) Community Hospital Scale. Our experience and lessons learned developing system-wide improvement and change in antimicrobial use might be useful to inform stewardship planning in Canada and other jurisdictions.

\section{Academic Hospital Foundation}

In 2009, Mount Sinai Hospital and University Health Network, two academic health science centres in Toronto, Ontario, jointly established a robust, well-resourced antimicrobial stewardship program (ASP). From the start, the hospital leadership at both institutions recognized the importance of antimicrobial stewardship as a patient safety imperative.

Our first foray into change management was prospective audit-and-feedback in intensive care units (ICUs): an infectious diseases physician and/or pharmacist would meet with the ICU teams on a daily basis, review antimicrobial practices on each patient, and provide coaching on best practices. Advice offered would include reducing use of unnecessarily broad-spectrum antimicrobials, limiting duration of therapy to those best supported by evidence, and emphasizing the need for source control where appropriate. The focus for the ASP was 
common infections managed by all physicians and not those that require consultation with an infectious diseases expert. Over the course of four years, we spread our program to five intensive care units, learned which change management practices worked and which did not, and leveraged our ICU successes to other areas of our hospitals. We identified the following two factors as critical to establishing ASPs in hospitals: strong leadership with clear accountability; and valid, reliable data to monitor progress.

\section{Strong leadership and clear accountability}

Dedicated individuals who led the program throughout the hospitals were key ingredients to the success of our ASP program. Although the most common model is an infectious diseases physician as leader, the physician does not need to be an expert in infectious diseases, and having a pharmacist as co-leader is often beneficial. In our experience, a pharmacist leading without a physician leader posed significant challenges. Our ASP leaders were respected clinicians, and they needed to have protected time with adequate salary support in order to carry out their ASP roles. We believe this initial investment was critical to our success.

For our ASP to be established effectively, it also required a champion from senior hospital leadership with credibility, authority, and a discretionary budget (2). For us, this leader was the Vice-President, to whom the ASP team was accountable. Traditionally, ASPs report to medical advisory committees (directly, or via pharmacy and therapeutics committees) or may be part of quality or infection control committees. Although these committees are important advocates for the work of ASPs, they may lack the authority and access to resources that ASPs require.

\section{Valid and reliable data}

Antimicrobial stewardship is a health care improvement initiative that often meets the "triple aims" of health care: it improves the quality of care, saves money, and improves the patient experience (3). Therefore, it must be laid upon a foundation of good data. In Canada, most hospital systems are designed to meet functional needs and do not lend themselves easily to the capture and retrieval of accurate and reliable data. Retrieving useable and meaningful data in a timely manner requires institutional investment. What we found initially was that the data were available, but the task of obtaining the data was left to individuals who neither had the expertise in these data systems nor the relationships with individuals who had such expertise. As a result, we needed to invest in getting the right person in place. Good data is necessary to assess progress. Although data problems can be complex, when appropriate resources were applied, solutions were achieved more easily than originally anticipated.

\section{Academic Hospital Spread}

In 2011, the Council of Academic Hospitals of Ontario awarded our ASP with a grant to implement antimicrobial stewardship programs in teaching hospitals with intensive care units. This was part of the Council's funding program Adopting Research to Improve Care (ARTIC), sponsored by the Ontario Ministry of Health and LongTerm Care. We worked with academic hospitals across the province to implement ASPs involving14 ICUs (including three pediatric ICUs). This "Antimicrobial Stewardship Programs in ICUs" project was based on what we learned from the successes and challenges we faced with our own program: the importance of strong leadership; clear accountability; clinical credibility; and a focus on obtaining timely, reliable and valid data.

The ARTIC ASP in ICUs project included coaching each hospital through the various stages of planning, implementing, evaluating and sustaining the establishment of an ASP. Each hospital identified an infectious diseases physician and a pharmacist who would serve as the subject matter experts and the ASP leads in their hospital. We found that antimicrobial stewardship expertise was not abundant in Ontario, which created a barrier for implementation. Many of the hospitals had to delay implementation to recruit or train subject matter experts. We recognized early on that there were knowledge gaps that needed to be addressed, even amongst experts in infectious diseases. Although all infectious diseases experts should be antimicrobial stewards, in fact, much of their practice is devoted to complex and rare infections. As a result they often do not have the opportunity to hone their expertise on the common infection syndromes that are at the heart of antimicrobial stewardship. We developed learning modules to address this knowledge gap and used whiteboard animation (short, scripted videos) to assist in the knowledge translation (4). We set clear reporting standards for the type of data we 
required (which included detailed antimicrobial usage and resistance data), and when it needed to be reported. It was important to ensure that each program was able to produce reliable and validated data in a timely manner.

We conducted on-site visits to each of the hospitals for a "project launch," and also carried out interviews with key stakeholders prior to each site implementing their ASP initiative. This was necessary to help understand the logistical, interpersonal and institutional challenges that each of the hospitals faced. We visited once more, approximately three months after ASP implementation, interviewed the stakeholders again, and provided the executive sponsor of the program and the ASP leaders with a report of our findings. These post-implementation visits not only assured us that milestones had been met but, more importantly, they allowed us to provide feedback to the executive sponsors. Several themes emerged from our visits, but invariably the advice we provided emphasized either: a) the need to improve the organizational structure to outline clear accountability; or b) the need for information technology support to improve timely and reliable data retrieval.

An important element to the ARTIC ASP in ICUs project was the development of antimicrobial measures for all high acuity ICUs in Ontario. Since 2008, Ontario's Critical Care Information System has been collecting real-time information on patients in Ontario's ICUs (5). Initially established to help identify ICU beds for critically ill patients, the amount and type of information contained in this information system has grown steadily. The ARTIC ASP in ICUs project team identified the opportunity to synergize with this information system early on. Working closely with Critical Care Services Ontario and CritiCall (the organization maintaining this information system), our project team helped introduce three new data fields: days of antibacterial therapy; days of antifungal therapy; and ICUonset $C$. difficile. Although we required the hospitals engaged in our project to report data above and beyond these newly created data fields, the new fields helped set a framework for antimicrobial stewardship data throughout hospitals in the province.

Upon the completion of the ARTIC ASP in ICUs project, all but one academic hospital in Ontario with an ICU had an established antimicrobial stewardship program, and each of these programs was able to report ICU-specific antimicrobial resistance and usage. Furthermore, all Level 2 and Level 3 ICUs in the province-regardless of their affiliation with academic health sciences centres-were able to readily access their ICU's days of antibacterial therapy, days of antifungal therapy, ICU-onset $C$. difficile infection, and even antimicrobial-free days. This makes Ontario the largest jurisdiction in the world (with over 200 ICUs, housing approximately 2,000 critical care beds) to be able to have real-time access to antimicrobial stewardship metrics for such a large number of patients. This set the stage for scaling antimicrobial stewardship to community hospitals.

\section{Community Hospital Scale}

In 2014, the Council of Academic Hospitals of Ontario partnered with Health Quality Ontario to fund a pilot program that would test the ability to "spread and scale" an ARTIC program. We were awarded this first-time grant for ASP development spread and scale.

Our community-based project used a "hub and spoke" model to demonstrate that the tools developed and the knowledge and expertise gained during the original project could be leveraged to help community hospitals implement ASPs without in-house infectious diseases specialists (Figure 1). We chose pharmacist and physician leaders from four established ASP "hub" sites (including one non-academic site) to each mentor approximately three community hospital "spoke" sites. Our ASP leaders offered guidance, but fundamentally the community program relied on the expertise of the hubs and the resourcefulness and dedication of the spokes. 
Figure 1: Hub and spoke model

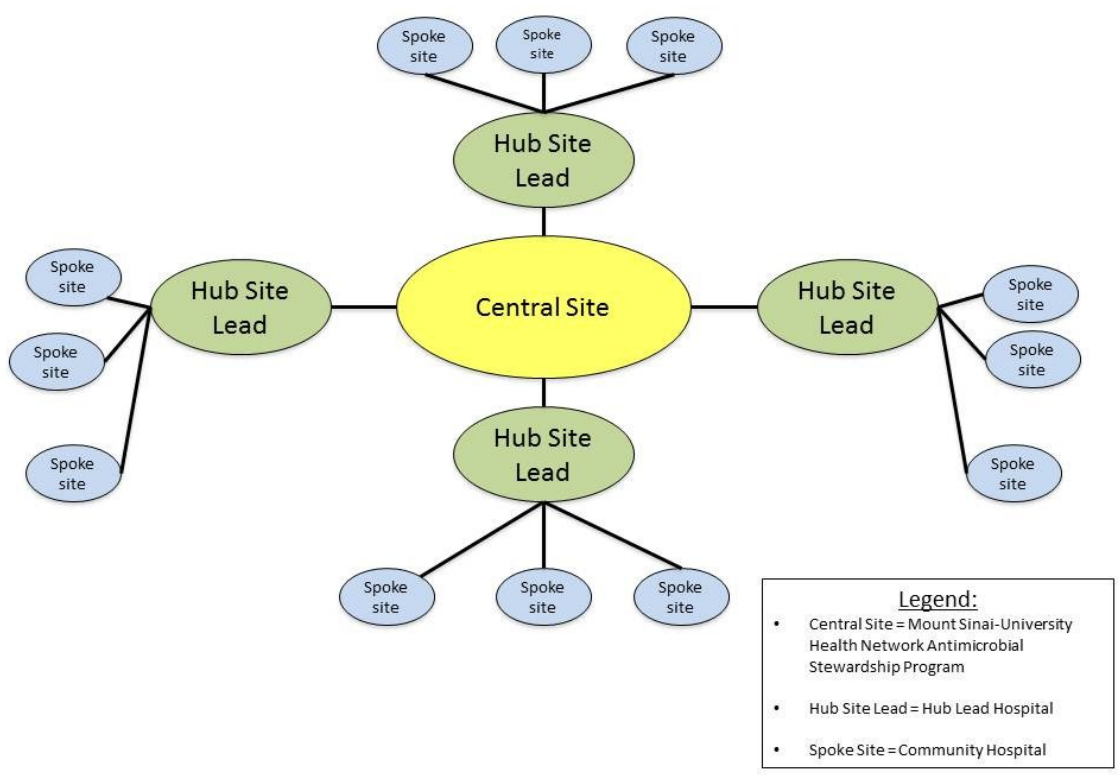

We are now four months into this community hospital project. Regional antimicrobial stewardship communities of practice are now becoming established. Informal observations and feedback have already been positive, instilling a high degree of confidence that the program will be successful.

\section{Conclusion}

In summary, we found that the support our team received at our two academic institutions, Mount Sinai Hospital and University Health Network, fostered the success of our ASP. Subsequently, a larger group of Ontario health care leaders have allowed antimicrobial stewardship to grow organically throughout a large Canadian province in only four short years. Ontario has now set a bar for antimicrobial stewardship implementation. This modelstarting with academic health sciences centres, and antimicrobial stewardship experts and leaders who are then supported to mentor and develop new experts and leaders-could be copied in other jurisdictions both within and outside of Canada.

\section{Acknowledgements}

We would like to acknowledge the various individuals who have demonstrated strong leadership and have supported the Mount Sinai Hospital-University Health Network Antimicrobial Stewardship Program over the years: Dr. Charlie Chan, Dr. Rupert Kaul, Dr. Conrad Liles, Scott Mclntaggart, Emily Musing, Dr. Gary Newton, Dr. Maureen Shandling, Dr. Tom E. Stewart, and Bill Wilson. Additionally, we are grateful for the continued support of Karen Michell and Michelle Grouchy (Council of Academic Hospitals of Ontario), and Lee Fairclough and Mark Robson (Health Quality Ontario). Finally, special thanks to all of our colleagues and collaborators who have helped bring antimicrobial stewardship to Ontario.

\section{Funding}

The Council of Academic Hospitals in Ontario ARTIC ICU in ASP project was supported by a grant from the Council of Academic Hospitals of Ontario and the Ontario Ministry of Health and Long-Term Care, which provided salary support to Dr. Yoshiko Nakamachi and Dr. Andrew Morris. The Council of Academic Hospitals of 
Ontario-Health Quality Ontario ARTIC CHILL project was supported by a grant from the Council of Academic Hospitals of Ontario and Health Quality Ontario, which provided salary support to Sarah West.

\section{Conflict of interest}

None

\section{References}

(1) Dellit TH, Owens RC, McGowan JE Jr., Gerding DN, Weinstein RA, Burke JP, et al. Infectious Diseases Society of America and the Society for Healthcare Epidemiology of America Guidelines for Developing an Institutional Program to Enhance Antimicrobial Stewardship. Clin Infect Dis. 2007;44(2):159-77.

(2) Morris AM, Stewart TE, Shandling M, Mclntaggart S, Liles WC. Establishing an antimicrobial stewardship program. Healthc Q. 2010;13(2):64-70.

(3) Berwick DM, Nolan TW, Whittington J. The triple aim: Care, health, and cost. Health Aff (Millwood). 2008;27(3):759-69.

(4) Dresser L, Biomedia K. Antimicrobial stewardship in critical care [whiteboard animation]. 2013. https://www.youtube.com/watch?v=-G4cEYQBVu4

(5) Ontario Ministry of Health and Long-Term Care-Critical Care Strategy. Critical Care Information System. 2012 Jun 25. http://www.health.gov.on.ca/en/pro/programs/criticalcare/ccis.aspx. 